\title{
Ribaucour type Transformations for the Hessian One Equation *
}

\author{
Antonio Martínez, Francisco Milán and Keti Tenenblat \\ Departamento de Geometría y Topología, Universidad de Granada, 18071 Granada. \\ Spain \\ e-mail: amartine@ugr.es \\ Departamento de Geometría y Topología, Universidad de Granada, 18071 Granada. \\ Spain \\ e-mail: milan@ugr.es \\ Departamento de Matemática, Universidade de Brasília, 70910-900 Brasília. \\ Brasil. \\ e-mail: K.Tenenblat@mat.unb.br

\begin{abstract}
We extend the classical theory of Ribaucour transformations to the family of improper affine maps and use it to obtain new solutions of the hessian one equation. We prove that such transformations produce complete, embedded ends of parabolic type and curves of singularities which generically are cuspidal edges. Moreover, we show that these ends and curves of singularities do no intersect. We apply Ribaucour transformations to some helicoidal improper affine maps providing new 3-parameter families with an interesting geometry and a good behavior at infinity. In particular, we construct improper affine maps, periodic in one variable, with any even number of complete embedded ends.
\end{abstract}

2000 Mathematical Subject Classification: 53A15

Keywords: Ribaucour transformations; Improper affine spheres; Hessian one equation.

\section{Introduction}

Differential geometry of surfaces and partial differential equations (PDEs) are tied up by a productive interaction by means of which both theories benefit mutually.

\footnotetext{
* Research partially supported by Ministerio de Educación Grants No: MTM2013-43970P, No: PHB2010-0109, Junta de Anadalucía Grants No. FQM325, N0. P06-FQM-01642. Ministério de Ciência e Tecnologia, CNPq Proc. No. 303774/2009-6. Ministério de Educação, CAPES/DGU Proc. No. 23038010833/2010-37.
} 
Actually, many classic PDEs are linked to interesting geometric problems. The geometry allows to integrate these equations, to establish non trivial properties of the solutions and to give some superposition principles which determine new solutions in terms of already known solutions.

One of the biggest contributions from geometry to PDEs theory is MongeAmpère equations. Such equations are totally non linear PDEs which model interesting geometric aspects related to the curvature, and its study has become a topic of great mathematical importance. Among the most outstanding MongeAmpère equations we can quote the hessian one equation:

$$
\operatorname{Det}\left(\nabla^{2} f\right)=1, \quad \text { in } \Omega .
$$

This is the easiest Monge-Ampère equation, and it appears in problems of affine differential geometry, minimal surfaces, flat surfaces and Calabi-Yau metrics, as well as in the description of area-preserving diffeomorphisms. The above equation has been studied from a global perspective by many authors, [20, $21,26]$. In particular, it is known that the only global $\mathcal{C}^{2}$ solutions of this equation are the quadratic polynomials $[4,7,18]$, and that the exterior Dirichlet problem has a solution $[6,12,13]$. The solutions defined on $\mathbb{R}^{2}$ minus a finite number of points are classified in [14], and a local classification result for isolated singularities is obtained in [1].

Another important issue in the theory of geometric PDEs is the study of singularities. Concerning equation (1.1), a geometric theory of smooth maps with singularities (improper affine maps) has been developed in [22]. Actually, improper affine maps are given locally as a pair $(\Omega, f)$ of a solution of (1.1), and they can be recovered in terms of their singular set. Generically, the singularities are cuspidal edges and swallowtails, (see [1, 17, 24, 25]).

Several methods as the method of perturbation, [28], integrable systems, [23] and Weierstrass' type representation, [1, 11, 22, 24] have been used in the construction of solutions of (1.1). But the classical theory of surfaces shows that geometric transformations may also be used to construct new surfaces from a given one. Recently, the third author and her collaborators (see $[8,9,10,19]$ ) have extended and applied a classical theory on Ribaucour transformations, developed by Bianchi in 1918-1919, [3], to provided global description of new families of complete minimal and constant mean curvature surfaces obtained from surfaces which are invariant under a one-parametric group of transformations.

In this work, we shall introduce a Ribaucour type transformation between improper affine maps, denoted by $\mathcal{R}$-transformation, that provides the description of a large class of new examples with an interesting geometry and a good behavior at infinity.

The paper is organized as follows. In Section 2, we recall some basic facts of the theory of improper affine maps and define the affine isothermic coordinates.

In Section 3, we introduce the notion of $\mathcal{R}$-transformation between such surfaces and show that $\mathcal{R}$-transformations produce embedded ends of parabolic type and curves of singularities which are, generically, cuspidal edges. Moreover we prove that these ends and curves of singularities do not intersect. 
In Section 4, we describe all improper affine maps obtained by applying an $\mathcal{R}$-transformation to some helicoidal improper affine maps. We also determine the ends of parabolic type and the curves of singularities produced by the $\mathcal{R}$ transformation.

\section{A complex resolution for the Hessian one equa- tion}

In this section, we recall some results from the theory of improper affine maps, which are surfaces in $\mathbb{R}^{3}$ locally given as vertical graphs of solutions of the Hessian one equation (1.1), that may have some admissible singularities. For more details see [5, 22].

Let $\Sigma$ be an orientable 2-manifold and consider $\psi: \Sigma \longrightarrow \mathbb{R}^{2} \times \mathbb{R}$ the improper affine map induced by a special Lagrangian immersion $\mathcal{L}:=x+\sqrt{-1} n$, $\mathcal{L}: \Sigma \longrightarrow \mathbb{C}^{2} \equiv \mathbb{R}^{2}+\sqrt{-1} \mathbb{R}^{2}$, where

$$
x: \Sigma \longrightarrow \mathbb{R}^{2}, \quad n: \Sigma \longrightarrow \mathbb{R}^{2},
$$

are differentiable maps. Then, $\psi$ may be written as

$$
\psi:=\left(x,-\int<n, d x>\right)
$$

and the non-regular points of $\psi$ correspond with the degenerate points of its flat fundamental form $d s^{2}$,

$$
d s^{2}:=<d x, d x>
$$

Because $\mathcal{L}$ is special and Lagrangian, we have that, around any regular point, $\psi$ is a vertical graph of a solution $f: \Omega \subseteq \Sigma \longrightarrow \mathbb{R}$ of the unimodular Hessian equation (1.1) and

$$
n=-\nabla f=-\left(f_{x_{1}}, f_{x_{2}}\right), \quad\left(x_{1}, x_{2}\right) \in \Omega .
$$

Thus, at the regular points, $\psi$ is an improper affine sphere with BerwaldBlaschke metric $g$ given by

$$
g:=-<d x, d n>.
$$

We say that the singularities of $\psi$ are admissible since they are not degenerate points of the induced metric $d \tau^{2}$,

$$
d \tau^{2}:=<d x, d x>+<d n, d n>.
$$

The metric $d \tau^{2}$ generates on $\Sigma$ the complex structure of a Riemann surface and we have the following Weirstrass' type representation:

Theorem 2.1. Complex Representation, [22]: 
- Let $\psi: \Sigma \longrightarrow \mathbb{R}^{2} \times \mathbb{R}$ be an improper affine map induced by the special Lagrangian immersion $\mathcal{L}:=x+\sqrt{-1}$. If we identify $\mathbb{R}^{2}$ to $\mathbb{C}$ in the standard way, then there exists a regular planar complex curve $\alpha:=(F, G): \Sigma \longrightarrow \mathbb{C}^{2}$ such that,

$$
\begin{aligned}
& x=G+\bar{F}, \quad n=\bar{F}-G \\
& \psi:=\left(G+\bar{F}, \frac{1}{2}\left(|G|^{2}-|F|^{2}\right)+\operatorname{Re}\left(G F-2 \int F d G\right)\right) .
\end{aligned}
$$

Moreover, the induced metric, the Berwald-Blaschke metric and the flat fundamental form are given, respectively, by

$$
\begin{aligned}
d \tau^{2} & =2\left(|d G|^{2}+|d F|^{2}\right), \\
g & =|d G|^{2}-|d F|^{2}, \\
d s^{2} & =|d G|^{2}+|d F|^{2}+d G d F+d \bar{G} d \bar{F} .
\end{aligned}
$$

- Conversely, if $\Sigma$ is a Riemann surface and $(F, G): \Sigma \longrightarrow \mathbb{C}^{2}$ a regular complex curve, then (2.7) gives an improper affine map which is well defined if and only if $\int F d G$ does not have real periods.

We shall call $(F, G)$ Weierstrass data of $\psi$.

Remark 1. From (2.7) and (2.8), the admissible singularities of $\psi$ correspond to the points where $|d F|=|d G| \neq 0$. Also, it is clear that $d \tau^{2}$ and $g$ are conformal metrics.

The $(2,0)$-part of $d s^{2}$ is a holomorphic quadratic differential which is globally defined and it can be written as

$$
Q=d G d F .
$$

This form $Q$ is called $\mathcal{L}$-Hopf differential and its zeros are the $\mathcal{L}$-umbilical points of $\psi$.

Coordinates in which both fundamental forms, $g$ and $d s^{2}$ diagonalize simultaneously give an $\mathcal{L}$-curvature lines parametrization (not necessarily conformal).

A conformal $\mathcal{L}$-curvature line parametrization is called affine isothermic and, in this case, the pre-images of the $\mathcal{L}$-curvature lines are the lines $u=$ const and $v=$ const, where $z=u+\sqrt{-1} v$ is a conformal coordinate.

Proposition 2.2. Let $\psi: \Sigma \longrightarrow \mathbb{R}^{2} \times \mathbb{R}$ be an improper affine map and $p \in \Sigma$ a non $\mathcal{L}$-umbilical point. Then, there is an affine isothermic parametrization of $\psi$ around of $p$.

Proof. Since $(d G d F)(p) \neq 0$, there exists a complex parameter $z=u+\sqrt{-1} v$ around $p$ such that

$$
d G d F=d z^{2} .
$$


From (2.8) and (2.10) we conclude that

$$
\begin{aligned}
d \tau^{2} & =4 \cosh (2 \phi)|d z|^{2}, \\
g & =2 \sinh (2 \phi)|d z|^{2}, \\
d s^{2} & =4 \cosh ^{2}(\phi) d u^{2}+4 \sinh ^{2}(\phi) d v^{2},
\end{aligned}
$$

where $\phi$ is the harmonic function given by

$$
\phi=\frac{1}{2} \log \left|\frac{d G}{d F}\right| .
$$

Remark 2. From Proposition 2.2 and having in mind that $x+\sqrt{-1} n$ is special and Lagrangian one can see that, at the regular points, the pair of quadratic forms $\left(g, d s^{2}\right)$ is a Codazzi pair with constant Gaussian curvature one,

$$
K\left(d s^{2}, g\right) \equiv 1
$$

Actually, around any non $\mathcal{L}$-umbilical point, the principal curvatures of the pair $\left(g, d s^{2}\right)$ are given by

$$
\lambda_{1}=-\tanh (\phi), \quad \lambda_{2}=-\operatorname{coth}(\phi) .
$$

Many other examples of Codazzi pairs also appear in $[2,16,27]$ and references therein.

Remark 3. From (2.8) and (2.12), the harmonic function $\phi$ vanishes at the admissible singularities of $\psi$. Moreover, the elliptic paraboloid is characterized by the fact that $\phi$ is a non vanishing constant, see $[4,11,18]$.

\section{$3 \quad \mathcal{R}$-transformations of improper affine maps}

Now, we can extend the classical theory of Ribaucour transformations to the family of improper affine maps in the following way.

Definition 3.1. Consider $\psi: \Sigma \longrightarrow \mathbb{R}^{2} \times \mathbb{R}$ and $\widetilde{\psi}: \widetilde{\Sigma} \longrightarrow \mathbb{R}^{2} \times \mathbb{R}$ two improper affine maps induced by the special Lagrangian immersions $\mathcal{L}:=x+\sqrt{-1} n$ and $\widetilde{\mathcal{L}}:=\widetilde{x}+\sqrt{-1} \widetilde{n}$. We say that $\psi$ and $\widetilde{\psi}$ are associated by an $\mathcal{R}$-transformation if there is a differentiable function $h: \Sigma \longrightarrow \mathbb{R}$ and a biholomorphism $\mathcal{R}: \Sigma \longrightarrow \Sigma$ such that

- $x(p)+h(p) n(p)=\widetilde{x}(\mathcal{R}(p))+h(p) \widetilde{n}(\mathcal{R}(p))$ for all $p \in \Sigma$.

- $\mathcal{R}$ preserves the $\mathcal{L}$-Hopf differential. 
Theorem 3.2. Two improper affine maps are associated by an $\mathcal{R}$-transformation if and only if they admit Weierstrass data, defined on the same Riemann surface $\Sigma$, such that either $(\widetilde{F}, \widetilde{G})-(F, G)$ is constant or there is a constant $c \in \mathbb{R}-\{0\}$ such that

$$
(\widetilde{F}, \widetilde{G})=\left(F+\frac{1}{c R}, G+R\right),
$$

where $R$ is a holomorphic solution of the following equivalent Riccati equations

$$
d R+d G=c R^{2} d F, \quad d G=c R^{2}\left(d F+d\left(\frac{1}{c R}\right)\right) .
$$

Proof. From (2.6), (2.9) and Definition 3.1, if we denote by $(F, G)$ and $(\widetilde{F}, \widetilde{G})$ the Weierstrass data of $\psi$ and $\widetilde{\psi} \circ \mathcal{R}$, respectively, then we have that either $\widetilde{F}-F$ and $\widetilde{G}-G$ are simultaneously constants or

$$
d F d G=d \widetilde{F} d \widetilde{G}, \quad \frac{\overline{\widetilde{F}}-\bar{F}}{R}=\frac{h-1}{h+1}=\frac{\widetilde{F}-F}{\bar{R}},
$$

with $R=\widetilde{G}-G$ a non-constant function, (note that $h$ is a real function).

Thus, the holomorphic function $R(\widetilde{F}-F)$ must be a non zero real constant and there exists $c \in \mathbb{R}-\{0\}$ such that

$$
R(\widetilde{F}-F)=(\widetilde{G}-G)(\widetilde{F}-F)=\frac{1}{c},
$$

which implies (3.1).

Now, by differentiating (3.4), $R$ satisfies the following differential equation

$$
d \widetilde{G}-d G=c R^{2}(d F-d \widetilde{F})=c R^{2}\left(1-\frac{d G}{d \widetilde{G}}\right) d F
$$

equivalent to (3.2), because $d R=d \widetilde{G}-d G$ has only isolated zeros.

On the other hand, from (3.3) and (3.4), we obtain

$$
h=\frac{c|R|^{2}+1}{c|R|^{2}-1}
$$

and the converse is clear.

Remark 4. $(\widetilde{F}, \widetilde{G})-(F, G)$ is constant if and only if the corresponding Lagrangian immersions $\widetilde{\mathcal{L}}$ and $\mathcal{L}$ are the same up to translation in $\mathbb{C}^{2}$.

Corollary 3.3. If $\psi$ and $\widetilde{\psi}$ are improper affine maps associated by an $\mathcal{R}$ transformation such that their Weierstrass data do not differ by a constant, then the function $h$ in Definition 3.1 is given by

$$
h=\left(\operatorname{coth}\left(\frac{\phi+\widetilde{\phi}}{2}\right)\right)^{\varepsilon},
$$

where $\varepsilon=1$ if $c>0, \varepsilon=-1$ if $c<0$, and $c$ is the non zero constant of Theorem 3.2. 
Proof. From (2.12), (3.1) and (3.2), we get

$$
\left|c R^{2}\right|=\left|\frac{d \widetilde{G}}{d F}\right|=\left|\frac{d G}{d \widetilde{F}}\right|=\left|\frac{d \widetilde{G} d G}{d F d \widetilde{F}}\right|^{1 / 2}=\exp (\phi+\widetilde{\phi})
$$

and (3.5) gives (3.6).

From [22], we know that each complete end of an improper affine map is biholomorphic to a punctured disk $\mathcal{D}-\left\{p_{0}\right\} \subset \mathbb{C}$ and the Weierstrass data extend meromorphically to the puncture $p_{0}$. Thus, we can determine when an $\mathcal{R}$-transformation produces new ends.

Theorem 3.4. Let $\widetilde{\psi}: \mathcal{D}-\left\{p_{0}\right\} \longrightarrow \mathbb{R}^{2} \times \mathbb{R}$ be an improper affine map associated by an $\mathcal{R}$-transformation to an improper affine map, $\psi: \mathcal{D} \longrightarrow \mathbb{R}^{2} \times \mathbb{R}$. Then $\widetilde{\psi}$ has an end at $p_{0} \in \mathcal{D}$ if and only if it is either a zero or a pole of the complex function $R$ given in Theorem 3.2.

In this case, if the $\mathcal{L}$-Hopf differential $Q$ of $\psi$ does not vanish at $p_{0}$, then the end of $\widetilde{\psi}$ at $p_{0}$ is complete, embedded and of parabolic type.

Proof. From (3.1) and having in mind that the movable singularities of $R$ are simple poles, it is clear that $\widetilde{\psi}$ has an end at $p_{0}$ if and only if $p_{0}$ is either a zero or a pole of $R$.

In this case, if $Q\left(p_{0}\right) \neq 0$, then (3.2) gives

$$
(d R)\left(p_{0}\right)=-(d G)\left(p_{0}\right) \neq 0, \quad\left(\text { resp. } d\left(\frac{1}{c R}\right)\left(p_{0}\right)=-(d F)\left(p_{0}\right) \neq 0\right)
$$

and $p_{0}$ is a simple zero (resp. a simple pole). Hence, see [11, 22], $\widetilde{\psi}$ has a complete embedded end at $p_{0}$ and the same behavior at infinity that the revolution improper affine map with Weierstrass data

$$
\left(\frac{1}{c\left(z-p_{0}\right)}, z-p_{0}\right), \quad \text { or } \quad\left(\frac{z-p_{0}}{c}, \frac{1}{z-p_{0}}\right) \text {. }
$$

In a similar way, we can determine when an $\mathcal{R}$-transformation produces new curves of singularities.

Theorem 3.5. Let $\psi, \widetilde{\psi}: \Sigma \longrightarrow \mathbb{R}^{2} \times \mathbb{R}$ be improper affine maps associated by an $\mathcal{R}$-transformation. Then the singular set $\widetilde{\mathcal{S}}$ of $\widetilde{\psi}$ is the nodal set of the harmonic function $\phi-\log \left(|c||R|^{2}\right)$, where $\phi$ is given by (2.12) and $c, R$ by Theorem 3.2. In particular, the new ends do not intersect $\widetilde{\mathcal{S}}$.

Moreover, in an isothermic parameter $z$ around a singular point $p_{0}$, the curves of singularities are given by

$$
\left|R^{\prime}+G^{\prime}\right|=\left|c R^{2} F^{\prime}\right|=1
$$

and $p_{0}$ is a cuspidal edge if and only if

$$
\operatorname{Im}\left(\frac{R^{\prime \prime}+G^{\prime \prime}}{R^{\prime}+G^{\prime}}\right)\left(p_{0}\right) \neq 0 .
$$


Proof. From Remark 1, we have that the admissible singularities of $\widetilde{\psi}$ satisfy

$$
|d \widetilde{F}|=|d \widetilde{G}| \neq 0 .
$$

Thus, from (2.12) and Theorem 3.2, we obtain

$$
c^{2}|R|^{4}=\left|\frac{d \widetilde{G} d G}{d F d \widetilde{F}}\right|=\left|\frac{d G}{d F}\right|=\exp (2 \phi),
$$

which proves the first part of the theorem.

Now, if we take an isothermic parameter $z=u+\sqrt{-1} v$ so that $d G d F=$ $d z^{2}=d \widetilde{F} d \widetilde{G}$, then

$$
G^{\prime} F^{\prime}=1=\widetilde{F}^{\prime} \widetilde{G}^{\prime},
$$

and since

$$
\left|\widetilde{G}^{\prime}\right|^{2}=\left|R^{\prime}+G^{\prime}\right|^{2}=\left|c R^{2} F^{\prime}\right|^{2}=\left|c^{2} R^{4} \frac{d F}{d G}\right|,
$$

we get that (3.9) is equivalent to (3.7).

Moreover, the singular set of $\widetilde{\psi}$ around $p_{0}$ is characterized by

$$
\left|\widetilde{F}^{\prime}\right|^{2}=\left|\widetilde{G}^{\prime}\right|^{2}=1=\widetilde{F}^{\prime} \widetilde{G}^{\prime} .
$$

Actually, $\widetilde{G}^{\prime}=\widetilde{\widetilde{F}^{\prime}}$ and $(2.6)$ give $d \widetilde{x}=2 \widetilde{G}^{\prime} d u$ and $(0,1)$ is the null direction at the singular points.

Finally, since the tangent vector to the curve $\widetilde{S} \equiv\left|\widetilde{G}^{\prime}\right|^{2}-1=0$ is $\left(-\widetilde{S}_{v}, \widetilde{S}_{u}\right)$, we conclude that $p_{0}$ is a cuspidal edge if and only if

$$
0 \neq\left|\begin{array}{cc}
0 & 1 \\
-\widetilde{S}_{v} & \widetilde{S}_{u}
\end{array}\right|\left(p_{0}\right)=\widetilde{S}_{v}\left(p_{0}\right)
$$

that is, one gets (3.8), (see also Remark 3.1 in [15]).

\section{New families of examples}

In this section, we will obtain new 3-parameter families of improper affine maps by applying the theory in the previous section to some helicoidal improper affine maps.

For this study we will denote by $\mathbb{A}_{0}\left(\mathbb{R}^{3}\right)$ the group of unimodular affine transformations whose differential preserves the vertical direction $e_{3}=(0,0,1)$ and we consider the 1-parameter subgroup $\mathcal{G}$ of $\mathbb{A}_{0}\left(\mathbb{R}^{3}\right)$ given by the composition of a vertical translation plus a rotation around $\left\langle e_{3}\right\rangle$.

Then, a $\mathcal{G}$-helicoidal surface, (invariant under $\mathcal{G}$ ), is generated by a suitable curve $\alpha: \mathrm{I} \longrightarrow \mathbb{R}^{2} \times \mathbb{R}$ as

$$
\psi(s, t)=\left(\begin{array}{ccc}
\cos (t) & -\sin (t) & 0 \\
\sin (t) & \cos (t) & 0 \\
0 & 0 & 1
\end{array}\right) \alpha(s)+\left(\begin{array}{c}
0 \\
0 \\
a_{3} t
\end{array}\right), \quad a_{3} \in \mathbb{R} .
$$


Theorem 4.1. Let $\psi: \Sigma \longrightarrow \mathbb{R}^{2} \times \mathbb{R}$ be an improper affine map with Weierstrass data $(F, G)$. Then $\psi$ lies on a $\mathcal{G}$-helicoidal surface if and only if $F G=-a^{2}$ for some constant $a \in \mathbb{C}$.

Proof. Assume $\psi$ is $\mathcal{G}$-helicoidal, then from (4.1) and by the identification of $\mathbb{C}$ to $\mathbb{R}^{2}$ in the standard way, $\psi$ may be written as

$$
\psi(s, t)=\left(\exp (\sqrt{-1} t)\left(\alpha_{1}(s)+\sqrt{-1} \alpha_{2}(s)\right), \alpha_{3}(s)+a_{3} t\right),
$$

for some suitable curve $\alpha:=\left(\alpha_{1}+\sqrt{-1} \alpha_{2}, \alpha_{3}\right)$. Thus, we have that

$$
n(s, t)=-\exp (\sqrt{-1} t) \frac{\alpha_{1}(s) \alpha_{3}^{\prime}(s)-a_{3} \alpha_{2}^{\prime}(s)+\sqrt{-1}\left(a_{3} \alpha_{1}^{\prime}(s)+\alpha_{2}(s) \alpha_{3}^{\prime}(s)\right)}{\alpha_{1}(s) \alpha_{1}^{\prime}(s)+\alpha_{2}(s) \alpha_{2}^{\prime}(s)},
$$

and from $(2.6), 4 G F=\overline{(x+n)}(x-n)$ is a holomorphic function that depends only on the variable $s$, which implies $F G=-a^{2}$, for some constant $a \in \mathbb{C}$.

Conversely, if $F G=-a^{2}$, we distinguish two cases:

Case I: $a=0$ and then $\psi$ is, trivially, a rotational surface lying on an elliptic paraboloid, (see Figure 1).

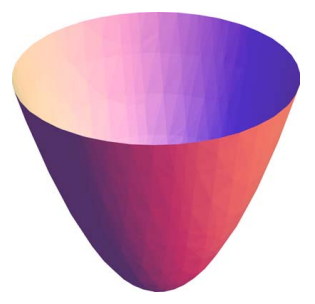

Figure 1: Elliptic paraboloid

Case II: $a \neq 0$ and then we can take $a=\exp \left(a_{1}+\sqrt{-1} a_{2}\right)$,

$$
G=a \exp (w), \quad F=-a \exp (-w),
$$

with $a_{1}, a_{2} \in \mathbb{R}$ and some holomorphic function $w=s+\sqrt{-1} t$. Actually, $s$ is the harmonic function $\phi: \Sigma \longrightarrow \mathbb{R}$ defined in (2.12).

Then, from (2.7), we have

$$
\begin{aligned}
\psi(s, t)= & \left(2 \exp (\sqrt{-1} t) \exp \left(a_{1}\right) \sinh \left(s+\sqrt{-1} a_{2}\right),\right. \\
& \left.\exp \left(2 a_{1}\right)\left(\sinh (2 s)+2 \cos \left(2 a_{2}\right) s-2 \sin \left(2 a_{2}\right) t\right)\right)
\end{aligned}
$$

and $\psi$ lies on a $\mathcal{G}$-helicoidal improper affine map generated by the rotation of the curve

$$
\alpha(s)=\left(2 \exp \left(a_{1}\right) \sinh \left(s+\sqrt{-1} a_{2}\right), \exp \left(2 a_{1}\right)\left(\sinh (2 s)+2 \cos \left(2 a_{2}\right) s\right)\right)
$$

and a vertical translation with $a_{3}=-2 \sin \left(2 a_{2}\right)$, which concludes the proof. 
Remark 5. In particular, $\psi$ is a rotational improper affine map if and only if $F G=-a^{2} \in \mathbb{R}$, (see Figures 1 and 2).
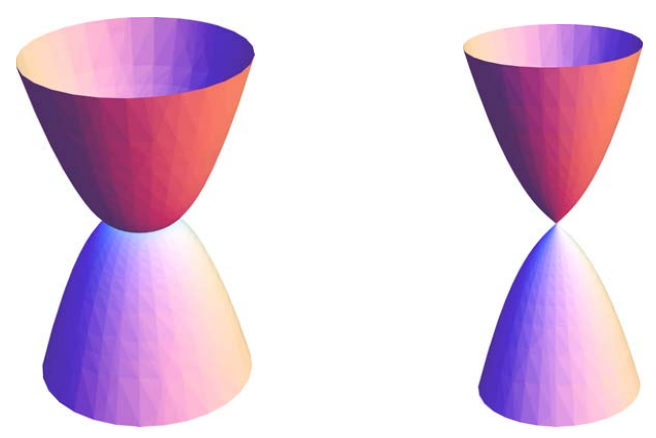

Figure 2: Rotational improper affine maps.

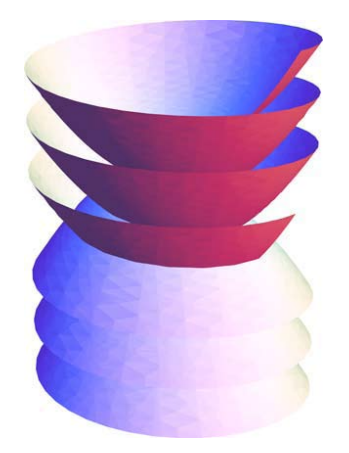

Figure 3: $\mathcal{G}$-helicoidal and non-rotational

Theorem 4.2. Let $\psi: \Sigma \longrightarrow \mathbb{R}^{2} \times \mathbb{R}$ be the $\mathcal{G}$-helicoidal improper affine map with the Weierstrass data $(F, G)$ given by (4.4), for some $a \in \mathbb{C}-\{0\}$. Then the family of improper affine maps $\widetilde{\psi}$ associated to $\psi$ by an $\mathcal{R}$-transformation is given by

$$
\widetilde{F}=-F \frac{1-b+(1+b) E}{1+b+(1-b) E}, \quad \widetilde{G}=G \frac{(b+1)^{2}+(b-1)^{2} E}{\left(b^{2}-1\right)(1+E)},
$$

with

$$
E=E(w)=k \exp (b w), \quad b=\sqrt{1+4 a^{2} c} \neq 0,
$$

where $k \in \mathbb{C}-\{0\}$ and $c \in \mathbb{R}-\{0\}$. 
Proof. From Theorem 3.2 and (4.4), we must consider the Riccati equation

$$
R^{\prime}(w)+a \exp (w)=a c R(w)^{2} \exp (-w) .
$$

Then, we eliminate the trivial solution $R=\exp (w) /(2 a c)$, when $1+4 a^{2} c=0$, because it gives $(\widetilde{F}, \widetilde{G})=-(F, G)$.

So, we have (4.7) from the general solution

$$
R=\frac{\exp (w)}{2 a c} \frac{1+b+(1-b) E}{1+E}=2 G \frac{1+b+(1-b) E}{\left(b^{2}-1\right)(1+E)}
$$

and

$$
\frac{1}{c R}=2 a \exp (-w) \frac{1+E}{1+b+(1-b) E}=-2 F \frac{1+E}{1+b+(1-b) E} .
$$

Theorem 4.3. Each improper affine map $\widetilde{\psi}$ in Theorem 4.2 has embedded complete ends of parabolic type determined by the points $w_{0}$ such that

$$
E\left(w_{0}\right)=-1, \quad \text { or } \quad E\left(w_{0}\right)=\frac{1+b}{b-1} .
$$

Moreover the singular set of $\tilde{\psi}$ is described by

$$
\frac{\exp (s)}{\left|b^{2}-1\right|}\left|\frac{1+b+(1-b) E}{1+E}\right|^{2}=1 .
$$

Proof. The first part is clear from Theorem 3.4, (4.4) and (4.9), since the $\mathcal{L}$-Hopf differential of $\psi$ is $Q=d G d F=a^{2} d w^{2} \neq 0$.

This also implies that $z=a w$ is an isothermic parameter and we deduce the second part from Theorem 3.5, (4.4) and (4.9).

In particular, when $4 a^{2} c+1=n^{2} / m^{2}$, with $n, m \in \mathbb{N}$, we have the following result, (see Figures 4 and 5).

Theorem 4.4. Let $\widetilde{\psi}$ be an improper affine map given by Theorem 4.2, with

$$
b=\frac{n}{m} \in \mathbb{Q}-\{0,1\}
$$

an irreducible rational number. Then

- $\widetilde{\psi}$ is periodic in $t$ with period $2 m \pi$.

- $\widetilde{\psi}$ has $2 n$ complete embedded ends of parabolic type.

- The singular set of $\widetilde{\psi}$ is contained in a compact set.

- There are two complete ends of geometric index $m$ given by the limit of $s$ to $\pm \infty$. 
Proof. In fact, $2 m \pi$ is the minimum common period of the terms $\exp (\sqrt{-1} t)$ and $\exp (\sqrt{-1} b t)$ in the Weierstrass data $(4.7)$ of $\widetilde{\psi}$.

Hence, we can consider $t \in[0,2 m \pi], b t \in[0,2 n \pi]$ and, from Theorem 4.3, the $2 n$ complete embedded ends of parabolic type of $\widetilde{\psi}$ are given by

$$
s_{1}+\sqrt{-1}\left(t_{1}+l \frac{2 \pi}{b}\right) \quad \text { and } \quad s_{2}+\sqrt{-1}\left(t_{2}+l \frac{2 \pi}{b}\right), \quad l=0, \ldots, n-1,
$$

where

$$
s_{1}+\sqrt{-1} t_{1}=\frac{1}{b} \log \left(\frac{-1}{k}\right) \quad \text { and } \quad s_{2}+\sqrt{-1} t_{2}=\frac{1}{b} \log \left(\frac{1+b}{k(b-1)}\right) .
$$

Moreover, from Theorem 3.4 and (4.9), $\widetilde{\psi}$ has two complete ends of geometric index $m$ when $s$ tends to $\pm \infty$, which do not intersect its singular set $\widetilde{\mathcal{S}}$, see Theorem 3.5 and (4.10).

Then, we conclude that $\widetilde{\mathcal{S}}$ is contained in a compact set.
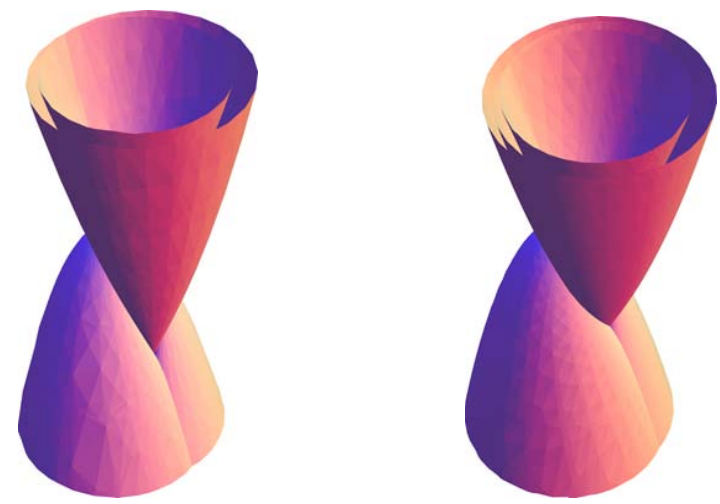

Figure 4: $\mathcal{R}$-transformations, $a=1, n=1, m=2$ and $n=1, m=3$ 

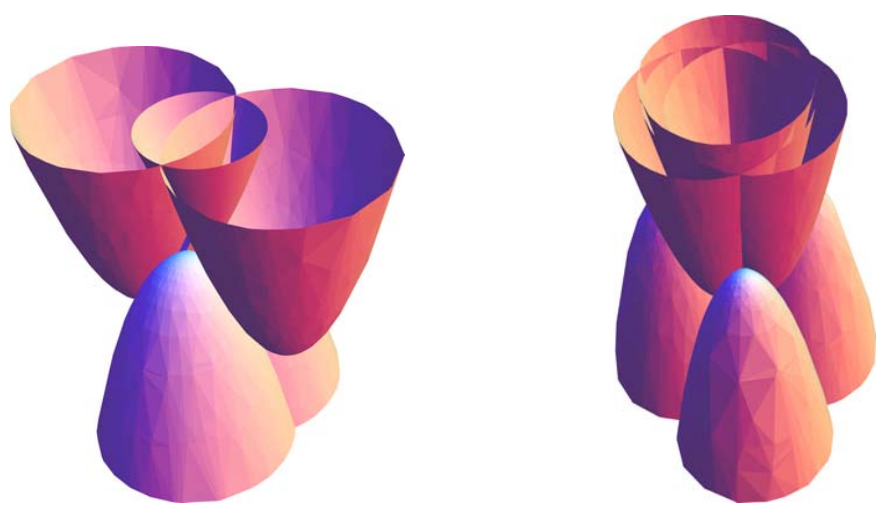

Figure 5: $\mathcal{R}$-transformations, $a=1, n=2, m=1$ and $n=3, m=2$

\section{References}

[1] J.A. Aledo, R.M.B. Chaves and J.A. Gálvez, The Cauchy Problem for Improper Affine Spheres and the Hessian One Equation, Trans. Amer. Math. Soc. 359 (2007), 4183-4208.

[2] J.A. Aledo, J.M. Espinar and J.A. Gálvez, The Codazzi equation for surfaces, Advances in Mathematics 224 (2010) 25112530.

[3] L. Bianchi, Lezioni di Geometria Differenziale, 2nd ed, Vol 2, 1903.

[4] E. Calabi, Improper affine hyperspheres of convex type and a generalization of a theorem by K. Jörgens, Michigan Math. J. 5 (1958), 105-126.

[5] E. Calabi, Affine differential geometry and holomorphic curves, Lect. Notes Math. 1422 (1990), 15-21.

[6] L. Caffarelli and Y. Li, An extension to a theorem of Jörgens, Calabi and Pogorelov, Comm. Pure Appl. Math. 56 (2003), 549-583.

[7] S.Y. Cheng and S.T. Yau, Complete affine hyperspheres. Part I. The completeness of affine metrics, Comm. Pure Appl. Math. 39 (1986), 839-866.

[8] A. Corro, W.P.Ferreira and K. Tenenblat, On Ribaucour Transformations for hypersurfaces, Matematica Contemporãnea, 17 (1999), 137-160.

[9] A. Corro, W.P.Ferreira and K.Tenenblat, Minimal Surfaces Obtained by Ribaucour Transformations, Geometriae Dedicata 96 (2003), 117-150.

[10] A. Corro, A. Martínez and K. Tenenblat, Ribaucour Transformations for flat surfaces in the hyperbolic 3-space, J. Math. Anal. Appl., 412 (2014), 720-743. 
[11] L. Ferrer, A. Martínez and F. Milán, Symmetry and uniqueness of parabolic affine spheres, Math. Ann. 305 (1996), 311-327.

[12] L. Ferrer, A. Martínez and F. Milán, An extension of a theorem by K. Jörgens and a maximum principle at infinity for parabolic affine spheres, Math. Z. 230 (1999), 471-486.

[13] L. Ferrer, A. Martínez and F. Milán, The space of parabolic affine spheres with fixed boundary, Monatsh. Math. 130 (2000), 19-27.

[14] J.A. Gálvez, A. Martínez and P. Mira, The space of solutions to the Hessian one equation in the finitely punctured plane, Journal de Mathématiques Pures et Appliquées 84 (2005), 1744-1757.

[15] T. Hoffmann, W. Rossman, T. Sasaki and M. Yoshida, Discrete flat surfaces and linear Weingarten surfaces in hyperbolic 3-space, Trans. Amer. Math. Soc. 364 (2012), 5605-5604.

[16] T. Klotz Milnor, Codazzi pairs on surfaces, Global differential geometry and global analysis, Proc. Colloq., Berlin 1979, Lect. Notes Math. 838, (1981), 263-274.

[17] G. Ishikawa and Y. Machida, Singularities of improper affine spheres and surfaces of constant Gaussian curvature, Internat. J. Math. 17(39) (2006), 269-293.

[18] K. Jörgens, Über die Lösungen der differentialgleichung $r t-s^{2}=1$. Math. Ann. 127 (1954), 130-134.

[19] M. Lemes, P. Roitman, K. Tenenblat and R. Tribuzy, Lawson correspondence and Ribaucour transformations. Trans. Amer. Math. Soc. 364 no. 12 (2012), 6229-6258.

[20] A.M. Li, F. Jia, U. Simon and R. Xu, Affine Bernstein problems and MongeAmpre equations, World Scientific 2010.

[21] A.M. Li, U. Simon and G. Zhao, Global affine differential geometry of hypersurfaces, Walter de Gruyter 1993.

[22] A. Martínez, Improper affine maps, Math. Z. 249 (2005), 755-766.

[23] M. Matsuda, Two methods of integrating Monge-Ampère equations, Trans. Amer. Math. Soc. 150 No1, (1970), 327-343.

[24] F. Milán, Singularities of improper affine maps an their Hessian equation, J. Math. Ann. Appl. 405 (2013), 183-190.

[25] F. Milán, The Cauchy problem for indefinite improper affine spheres and their Hessian aquation, Advances in Mathematics 251 (2014), 22-34. 
[26] K. Nomizu and T. Sasaki, Affine differential geometry, Cambridge University Press 1994.

[27] V. Oliker and U. Simon, Codazzi tensors and equations of MongeAmpre type on compact manifolds of constant sectional curvature, J. Reine Angew. Math. 342 (1983), 35-65.

[28] V. Oliker and P. Waltman, On the Monge-Ampère equation arising in the reflector mapping problem, Inst. for Mathematics and its Applications University of Minnesota, 1985. 